\title{
Ergodic crossover in partially self-avoiding stochastic walks
}

\author{
Juliana M. Berbert* \\ Instituto de Física Teórica (IFT), Universidade Estadual Paulista-UNESP, Caixa Postal 70532-2, Cep 01156-970 São Paulo, SP, Brazil
}

Rodrigo Silva González ${ }^{\dagger}$

Instituto de Ciências Exatas e Tecnológicas, Universidade Federal de Viçosa (UFV), Rodovia MG-230 Km $738810-000$, Rio Paranaíba, MG, Brazil

Alexandre Souto Martinez $z^{\ddagger}$

Faculdade de Filosofia Ciências e Letras de Ribeirão Preto (FFCLRP), Universidade de São Paulo (USP), Avenida Bandeirantes, 3900 14040-901, Ribeirão Preto, SP, Brazil and National Institute of Science and Technology in Complex Systems (LNCT-SC)

(Received 14 May 2013; published 11 September 2013)

\begin{abstract}
Consider a one-dimensional environment with $N$ randomly distributed sites. An agent explores this random medium moving deterministically with a spatial memory $\mu$. A crossover from local to global exploration occurs in one dimension at a well-defined memory value $\mu_{1}=\log _{2} N$. In its stochastic version, the dynamics is ruled by the memory and by temperature $T$, which affects the hopping displacement. This dynamics also shows a crossover in one dimension, obtained computationally, between exploration schemes, characterized yet by the trajectory size $\left(N_{p}\right)$ (aging effect). In this paper we provide an analytical approach considering the modified stochastic version where the parameter $T$ plays the role of a maximum hopping distance. This modification allows us to obtain a general analytical expression for the crossover, as a function of the parameters $\mu, T$, and $N_{p}$. Differently from what has been proposed by previous studies, we find that the crossover occurs in any dimension $d$. These results have been validated by numerical experiments and may be of great value for fixing optimal parameters in search algorithms.
\end{abstract}

DOI: 10.1103/PhysRevE.88.032119

\section{INTRODUCTION}

Deterministic walks in regular and disordered environments may present several interesting results [1-4]. For instance, consider a deterministic partially self-avoiding walk [5-8] in which a walker can visit $N$ sites, randomly distributed on a landscape, following the deterministic rule of going to the nearest site not visited in the last $\mu$ steps, including the current site [5]. One can compare this walker with a tourist exploring cities, and the memory $\mu$ with the time required for a city already visited to become attractive again to the tourist. In foraging, it may be important to avoid recently visited sites but allow returning after a time period $\mu$ (e.g., after a suitable time delay necessary for replenishment of exhaustible food resources) [9-16].

In one-dimensional systems, this dynamics has a crossover in the exploration behavior according to $\mu_{1}=\log _{2} N$. We have shown [8] that if $\mu<\mu_{1}$, the walker is trapped in small regions. Nevertheless, if $\mu \geqslant \mu_{1}$, the walker crosses the medium, if leaving from one extremity [8]. As an optimization problem, for small memories, compared to system size, one guarantees the whole landscape exploration $[8,17,18]$. The robustness of this crossover has been addressed with the inclusion of a stochastic factor.

If movements are according to a cost function dependent on $\mu$ and on a formal temperature $T$, then hops to nearest sites are favored $(T \rightarrow 0)$ while long jumps are also allowed

\footnotetext{
*berbertj@gmail.com

${ }^{\dagger}$ rodrigo.gonzalez@ufv.br

‡asmartinez@usp.br
}

PACS number(s): 05.40.-a, 05.70.Fh, 02.50.-r, 05.10.Gg

$(T \rightarrow \infty)$ [19-21]. In this scenario there is a nontrivial exploration crossover dependent on $\mu$ and $T$ [21].

The results of these numerical simulation are valid only for one-dimensional systems. To achieve an analytical method also valid for high dimensionalities, as demanded in optimization procedures, further simplifications are necessary. In this paper the walker also avoids the last $\mu$ visited sites, but its decision rules are limited to include only the walker's surroundings, which size is defined by the stochastic parameter $T$. Thus, the walker's reach is defined by $T$.

One can view this dynamics as a stochastic optimization algorithm that combines local and global exploration. If information is feeded to the system as it evolves, one cannot use traditional methods such as simulated annealing [22,23] or genetic algorithm [24], since the whole system to be optimized must be known beforehand. Here we address the situation where the information about the system is fed as it evolves, and only local information in the $T$ range must be supplied. To reach the best results, one can avoid the initial parametrization working along a crossover line between two regimes: a nonergodic one, which explores locally the parameter space, and the ergodic one, which drives the system away from the local minima. In this paper we present a model that has this kind of exploration crossover even for systems with arbitrary dimensions. In addition, our model allows us to obtain analytically the relation between the parameters $\mu$ and $T$ along the crossover line.

Our arguments are presented as follows. In Sec. II we describe the model and justify the simplifying assumptions. The analytical solution and numerical simulation method are discussed in Sec. III. We show that aging must be considered in our analytical approach to be compatible with computer 
simulations. In Sec. IV we present the data collapse of computer simulations and analytical treatment indicating a subtle change in the exploration scheme, transitioning from a nonergodic to an ergodic regime. Finally, in Sec. V we conclude, indicating systems where our model could be applied.

\section{MODEL}

Consider walks on a disordered landscape composed of $N$ sites. This landscape can represent, for example, localized feeding sites as flowers, trees, water holes, islands, etc. [5,20]. The disordered medium can be defined with each site coordinate, $x_{i}^{(k)}, i=1,2, \ldots, N$ and $k=1,2, \ldots, d$, being drawn in accordance with a random and uniform distribution along the edges of a $d$-dimensional hypercube. For a $d$-dimensional hypercube with edge length $L$, the mean site distance $\ell$ is the edge length of an also $d$-dimensional hypercube associated to each site. If these hypercubes do not overlap, $N \ell^{d}=L^{d} \Rightarrow$ $\ell=L N^{-1 / d}$. The mean site distance $\ell$ is used to normalize the distance between sites. To hop from one site to another, the walker uses a strategy based on some arbitrary cost function $E\left(D_{j, i}\right)$, which is a monotonically increasing function of the normalized Euclidean distance

$$
D_{j, i}=\frac{N^{1 / d}}{L}\left\{\sum_{k=1}^{d}\left[x_{i}^{(k)}-x_{j}^{(k)}\right]^{2}\right\}^{1 / 2}
$$

between sites $i$ and $j$. In the thermodynamical limit, $N \rightarrow$ $\infty$, this procedure preserves the constant site density as the system dimensionality varies and makes it possible to compare systems with distinct dimensionalities.

To move on this landscape, the walker strategy based in the cost function $E\left(D_{j, i}\right)$ follows a probability density function (PDF) that depends on the inverse of the formal temperature, $T>0$, and the walker memory, $\mu \geqslant 0$. The memory generates a self-avoiding window with the last $\mu$ visited sites, including the current site, which are forbidden revisitation in the next $\mu$ steps. The inverse of a formal temperature $T$ is the stochastic parameter $\beta=1 / T$, and as $\beta$ decreases, long hops are favored. Thus, the PDF is $W_{j \leftarrow i}=e^{-\beta E\left(D_{j, i}\right)} / \sum_{k=1}^{\prime N} e^{-\beta E\left(D_{k, i}\right)}$, and the summation $\sum^{\prime}$ excludes the forbidden sites by $\mu$. The denominator is the normalization factor $Z_{i}^{(\beta, \mu)}$. This model, named the stochastic tourist walk (STW), privileges local exploration when $T \rightarrow 0(\beta \rightarrow \infty)$ and extended exploration when $T \rightarrow \infty(\beta \rightarrow 0)$. By means of Monte Carlo numerical simulation, it has been shown a crossover between these localized and extended explorations that occurs for specific values of $T$ and $\mu$ [21].

For an analytical approach, in the particular case of the STW, the main difficulty concerns the normalization coefficient, which value changes as a function of the walker position. To circumvent this problem, we decided to modify the model. At each discrete time step, a walker moves on this medium hopping from one site to another site not visited in the $\mu$ previous steps, including the presently occupied site, and within a range $T$. The range $T \in\left(0, d^{1 / 2} / 2\right)$ defines the walker's maximum step length and a hypersphere centered in

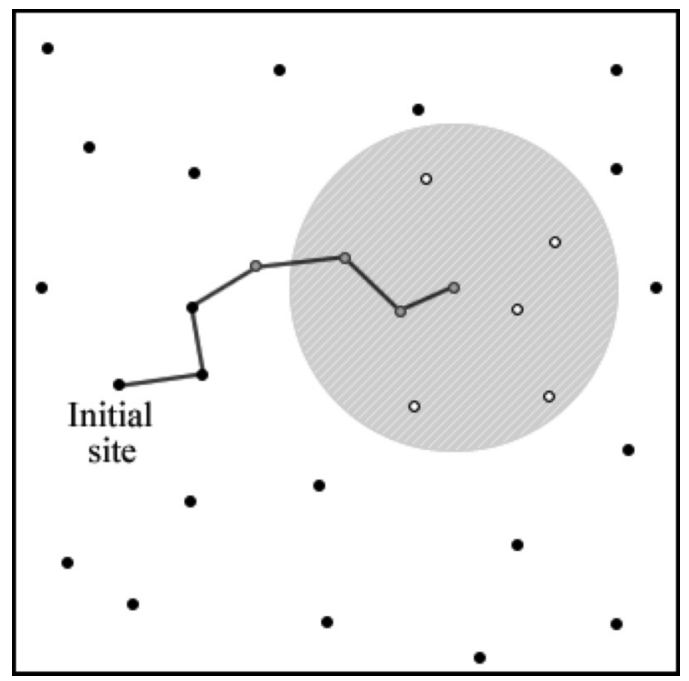

FIG. 1. Standard medium configuration for $d=2, N=30$, and $\mu=4$. Dots represent the sites. The line segments stand for the trajectory. The circle indicates the volume $V_{d}$ with $n_{v}=8$ and $n_{\mu}=3$ sites. Black dots are sites unreachable in the following step. Gray dots belong to the memory. White dots are allowed to be visited with probability $W=1 / 5$.

its position with volume

$$
V_{d}=\frac{\pi^{d / 2} T^{d}}{\Gamma(1+d / 2)},
$$

where $\Gamma(x)$ is the gamma function. This volume contains $n_{v}$ sites, out of which $n_{\mu} \leqslant \mu$ belong to the avoidance window, due to the memory $\mu$. For each step, the medium has at most $\mu$ sites forbidden to revisitation, $N-n_{v}$ sites (outside $V_{d}$ ) unreachable, and for the following step there are $n_{v}-n_{\mu}$ sites equally likely to be visited. The sketch in Fig. 1 illustrates this issue.

The probability of a walker to hop from site $i$ to $j$ is

$$
W_{j \leftarrow i}=\left\{\begin{array}{cl}
\left(n_{v}-n_{\mu}\right)^{-1} ; & \text { for } D_{i j} \leqslant T \text { and } j \notin n_{\mu}, \\
0 ; & \text { otherwise, }
\end{array}\right.
$$

where $D_{i j}$ is given by Eq. (1). As $n_{\mu}$ approaches $n_{v}$ in the volume $V_{d}$, fewer sites become available due to the avoidance window. Thus, the probability of each allowed site to be visited increases in the following step. However, if $\mu \geqslant n_{v}$ and $n_{\mu}=n_{v}$, then $W_{j \leftarrow i}$ diverges, because all sites inside $V_{d}$ are prohibited since they have already been visited in previous steps. The walker becomes trapped. To use the expression "survival probability" within the random walk context, we may say the walker dies when it is not able to reach any other site. This situation also occurs for the deterministic tourist walk with $\mu=N$ [5]. Notice that these trappings do not occur in the previously studied partially self-avoiding stochastic walks [21]. Contrary to these cases, where the hopping range has not been strictly defined (it is an emerging characteristics of the exponential function in the Boltzmann-Gibbs weight), here we abandon the Boltzmann-Gibbs weight and strictly fix the range, eventually producing traps.

According to the mean distance between sites, $\ell$, for $T<\ell$ and $\mu>0$, the walker is always trapped. For $T \geqslant \ell$, the walker survival depends on $\mu, n_{\mu}$, and $n_{v}$ : (1) if $n_{v}>\mu$, there are 
always allowed sites and the walker survives, (2) if $n_{v} \leqslant \mu$ and $n_{\mu}<n_{v}$, there are allowed sites and the walker survives, and (3) if $n_{v} \leqslant \mu$ and $n_{\mu}=n_{v}$, all volume $V_{d}$ is forbidden, leading to the walker's death. For $\mu=0$, the walker does not remember the presently occupied site, and thus it always survives because it can stay at the current site, regardless of the size of $T$.

To explore efficiently the random medium, the walker must survive as long as possible. In addition, as in the onedimensional cases for deterministic partially avoiding walks [17] and stochastic walks [21], there is a transition in the walker behavior, namely, a crossover between ergodic and nonergodic exploration regimes. The walker survival probability is the order parameter for this situation and depends on the number of allowed sites $n_{d}=n_{v}-n_{\mu}$, which depends on $T$ and $\mu$.

The following analysis is based on the volume $V_{d}$, where $d$ and $T$ are implicitly considered. Therefore, as we show, the exploration crossover occurs for an arbitrary $d$, not only for $d=1$, as described in Refs. [17,21].

\section{ANALYTICAL AND COMPUTATIONAL METHODS}

In this section we describe the analytical treatment and its validation by computational simulations.

\section{A. Analytical method}

The analytical treatment is based on the discrete random variable statistics. The number of sites $n_{v}$ inside the volume $V_{d}$ is a discrete random variable with mean value $\left\langle n_{v}\right\rangle=N v$, where $v=V_{d} / V^{\prime}$, with $V_{d}$ given by Eq. (2), and a $d$-dimensional volume $V^{\prime}$, with $N$ sites uniform and randomly drawn. It follows that $n_{v}$ is distributed according to a binomial, with success probability $v$. This result has been numerically validated (not shown). For $V^{\prime}=1$ and $v=V_{d}$, the $n_{v}$ distribution is

$$
p\left(n_{v}\right)=\left(\begin{array}{l}
N \\
n_{v}
\end{array}\right) V_{d}^{n_{v}}\left(1-V_{d}\right)^{N-n_{v}} .
$$

Since this is a static medium characteristic, $n_{v}$ is independent of $\mu$.

As is $n_{v}, n_{\mu}$ is also a random variable. To obtain its distribution, consider $n_{\mu}$ as the number of sites in $V_{d}$ that have a given characteristic $C$ and $M$ is the subset of all sites with this characteristic; see the Venn diagram in Fig. 2. The $n_{\mu}$ distribution is a hypergeometric distribution [25],

$$
p\left(n_{\mu}\right)=\left(\begin{array}{l}
n_{v} \\
n_{\mu}
\end{array}\right)\left(\begin{array}{l}
N-n_{v} \\
\mu-n_{\mu}
\end{array}\right)\left(\begin{array}{l}
N \\
\mu
\end{array}\right)^{-1} .
$$

This result has also been numerically validated (not shown).

If the condition $n_{v}=n_{\mu}$ is satisfied, the walker dies. Let $A$ be the event $n_{v}=k$ and $B$ be the event $n_{\mu}=k$. Although $n_{\mu} \leqslant n_{v}$, the value $n_{\mu}$ does not depend on $n_{v}$, but rather on $\mu$. Only if $\mu \geqslant n_{v}, n_{\mu}$ is equal to $n_{v}$. As $\mu$ is independent of $n_{v}$, the events $A$ and $B$ are independent. Therefore,

$$
P(A \cap B)=\left(\begin{array}{l}
\mu \\
k
\end{array}\right) V_{d}^{k}\left(1-V_{d}\right)^{N-k},
$$

where $V_{d}$ is given by Eq. (2).

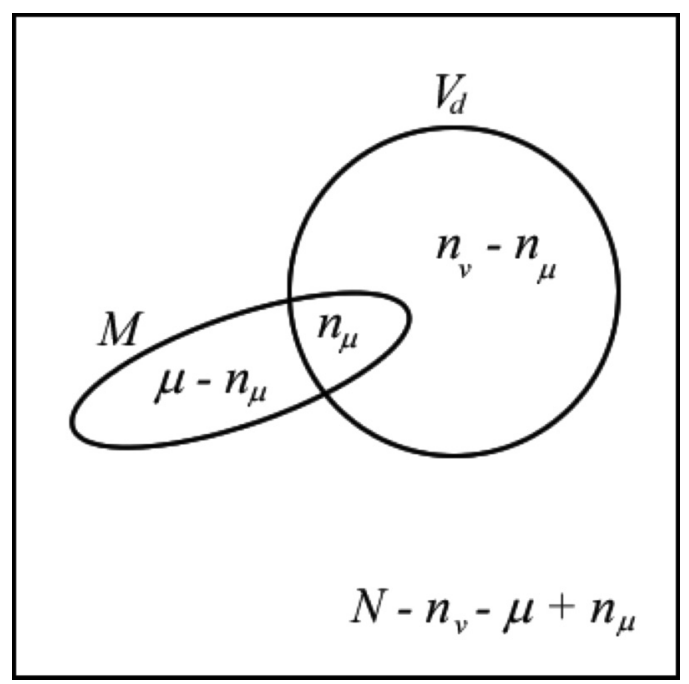

FIG. 2. In the Venn diagram, the intersection of $V_{d}$ and $M$ has $n_{\mu}$ sites. The $M$ subset not in $V_{d}$ has $\mu-n_{\mu}$ sites, and the subset $V_{d}$ not in $M$ has $n_{v}-n_{\mu}$ sites. The total amount of sites not in $M$ or in $V_{d}$ is $N-n_{v}-\mu+n_{\mu}$.

To describe the model dynamics, the relevant quantity to consider is the walker death probability $P_{\delta}$. It is defined at each step by the summation over all possible values of $k$ in Eq. (6). For $T$ large enough to allow a walk in this medium, the possible values of $k$ are in the interval $2 \leqslant k \leqslant \mu$. Note that, the case $k=0$ does not occur (there always is at least one site inside $V_{d}$ ), and for the case $k=1$ there is no walk. Thus, at each step, the walker death probability is

$$
\begin{aligned}
P_{\delta}\left(V_{d}, \mu\right) & =\sum_{k=2}^{\mu}\left(\begin{array}{l}
\mu \\
k
\end{array}\right) V_{d}^{k}\left(1-V_{d}\right)^{N-k} \\
& =\left(1-V_{d}\right)^{N-\mu}\left[1-\left(1-V_{d}\right)^{\mu}\left(1+\frac{\mu V_{d}}{1-V_{d}}\right)\right] .
\end{aligned}
$$

Figure 3 depicts Eq. (7) for a system with $N=100$ sites. The walker death occurs when $\mu \sim O(N)$, except when $V_{d} \rightarrow$ 0 . In this case, death occurs only if $n_{v}=1$; however, if there is a first step in the walk, $n_{v}>n_{\mu}$ and the survival is guaranteed. In the other cases, the walker survival always occurs.

One should note that Eq. (7) has a factor dependent only on the maximum number of available sites $N-\mu$, explicating the system finite size effect. The factor inside the brackets depends only on $\mu$ related to the memory effect. In the thermodynamics limit $(N-\mu \rightarrow \infty), P_{\delta} \rightarrow 0$, and the walker survival always occurs. Indeed, in this limit, if $V_{d}>0,\left\langle n_{v}\right\rangle=N V \rightarrow \infty$, and the Eq. (4) becomes $p\left(n_{v}\right)=\delta_{n_{v}, \infty}$. Thus, $n_{\mu}<n_{v}, \forall \mu \neq N$, favoring the walker survival. The plots in Fig. 3 illustrate the behavior of Eq. (7).

\section{B. Monte Carlo simulations}

To validate Eq. (7), we have performed computational simulations on disordered environments with $N=32,64$, and 128 sites and dimensionality $d=1,2,3$. We have varied the memory as $\mu=\{0 ; 1 ; 2 ; \ldots ; N\}$, and the volume as $V_{d}=\{0 ; 0.01 ; 0.02 \ldots ; 1\}$. Further, for each set of $N$ and $d, 10^{4}$ maps have been generated. On each map, $N$ walks have 


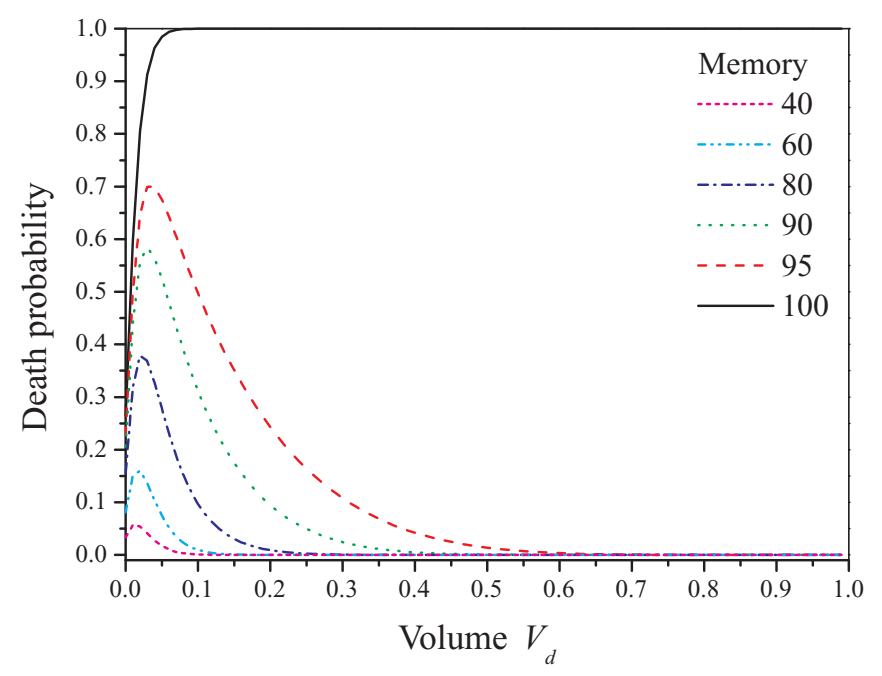

(a)

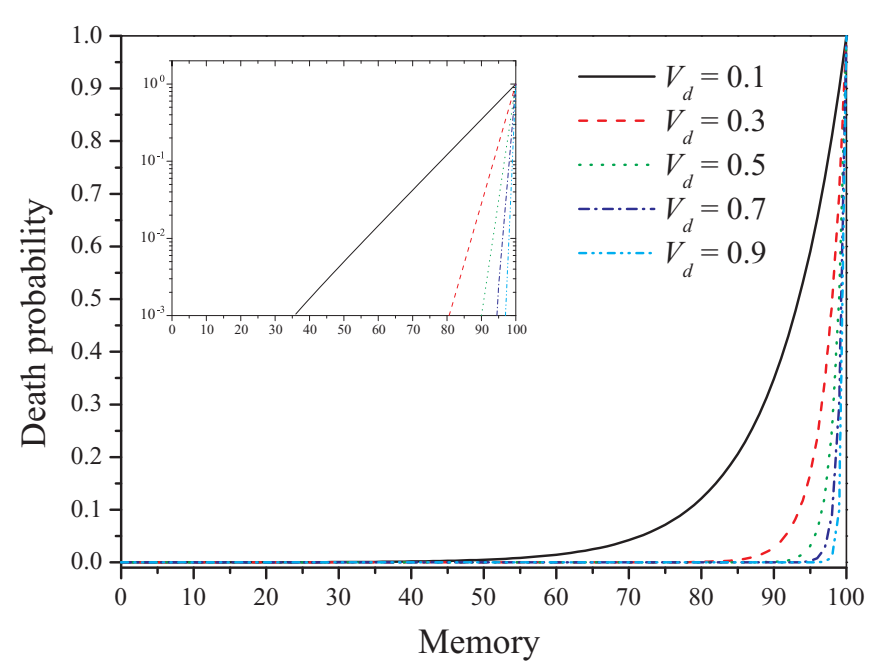

(b)

FIG. 3. (Color online) Walker death probability [Eq. (7)] for $N=$ 100 sites. (a) Constant $\mu$ and (b) constant $V_{d}$. Inset: The exponential behavior of the later case.

been performed, each walk starting from each site of the map. Therefore, there are $10^{4} \mathrm{~N}$ trajectories to analyze. For each trajectory, the walker explores the medium randomly going to one of the $n_{v}-n_{\mu}$ allowed sites, given by Eq. (3). After each step, the avoidance window, with at most $\mu$ sites, is updated.

Unlike the deterministic walks that finish in cycles, this walk does not end naturally, except when the walker dies. For this reason, it is necessary to limit the simulation time with a stop condition. Thus, we introduce a new variable, the exploration time $N_{p}$, which is the number of steps along the walker trajectory. The insertion of this variable has only a numerical justification, yet, as shown later, physically it generates the aging effect. Each trajectory has at most $N_{p}$ steps, which value is set a priori. Therefore, the trajectory ends when the walker dies or after $N_{p}$ steps, whichever occurs first. We have varied this parameter as $N_{p}=N, 5 N, 10 N, 25 N, 50 N, 70 N, 90 N$, and $100 N$ steps.

As in the analytical treatment, here we define the numerical walker death probability $P_{\delta}$. It is obtained by the ratio between the total number of walks that ended due to the walker's getting stuck and total number of walks.

The finite size effect is also observed in the computational simulations, by reducing the total death region as $V_{d} \rightarrow 0$. In addition, another effect appears, the boundary effect.

The boundary effect does not influence the analytical result because, hypothetically, the population of sites is uniform in all analyzed space regions, so that sites near the edges have the same number of neighbors as those closer to the center. In the numerical simulation, however, this is not true, since the sites closer to the edges have fewer neighbors than those in the center. Thus, the volume $V_{d}$ centered in sites closer to the edges has a nonpopulated area beyond the edges, violating the hypothesis of uniformity in all space regions and, therefore, compromising the simulation and increasing the death probability near the edges. Moreover, the walker death probability increases as $d$ increases with $N$ kept constant, contradicting the analytical result, according to which this probability does not depend explicitly on $d$. This contradictory increasing on the walker death probability is also explained by the boundary effect, which gets stronger as the system dimension increases.

A commonly used solution to fix the boundary effect is the use of periodic boundary conditions. Nevertheless, as a side effect, periodic boundary conditions also simulate an environment of infinite population $(N \rightarrow \infty)$, eliminating the finite size effect, which is indeed taken into account in analytical resolution. Further, using the thermodynamic limit $(N \rightarrow \infty)$, the survival is the general rule. Therefore, to observe the crossover between the walker's survival and death, it is not interesting to eliminate the finite size effect in the computational simulations, which prevents the use of periodic boundary conditions and therefore maintains the boundary effect in the system.

\section{TRANSITIONS TO ERGODICITY}

For a given set of parameters, the walker can perform a localized (nonergodic) or an extended (ergodic) exploration. The walker must survive as long as possible to increase

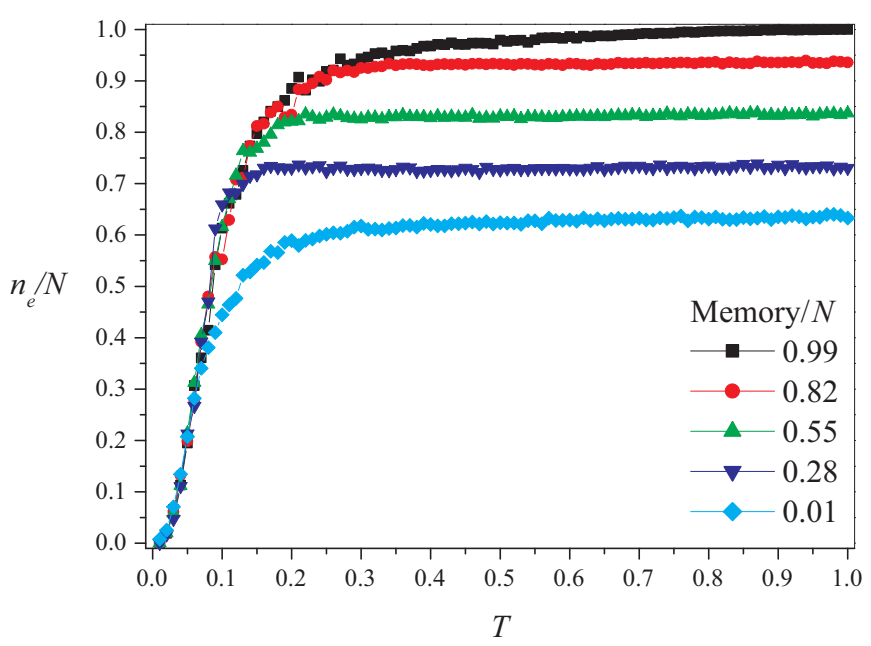

FIG. 4. (Color online) Visitation rate as function of $T$ and memory for $N=128, d=1$, and $N_{p}=N$. 
the medium exploration. Therefore, the crossover is obtained by the walker survival probability. Nevertheless, prolonging the walker survival makes the system undergo an aging phenomena.

\section{A. Exploration crossover}

To verify that high walker survival is a guarantee of high exploration, computational experiments have been performed measuring the visitation rate (ratio between the number of distinctly visited sites, $n_{e}$, and $N$ ) as a function of $T$ and $\mu$. Figure 4 shows the obtained results for $N=128, d=1$, and $N_{p}=N$, which is the lowest number of steps required for a total visitation. It is worth mentioning that this behavior is the same for other dimensions, and similarly for the walker death probability, the visitation rate does not depend strongly on $d$. Further, for $V<1$, all sites are visited even for $T=1$, which is equal or smaller than $T_{\max }=\sqrt{d} / 2$.

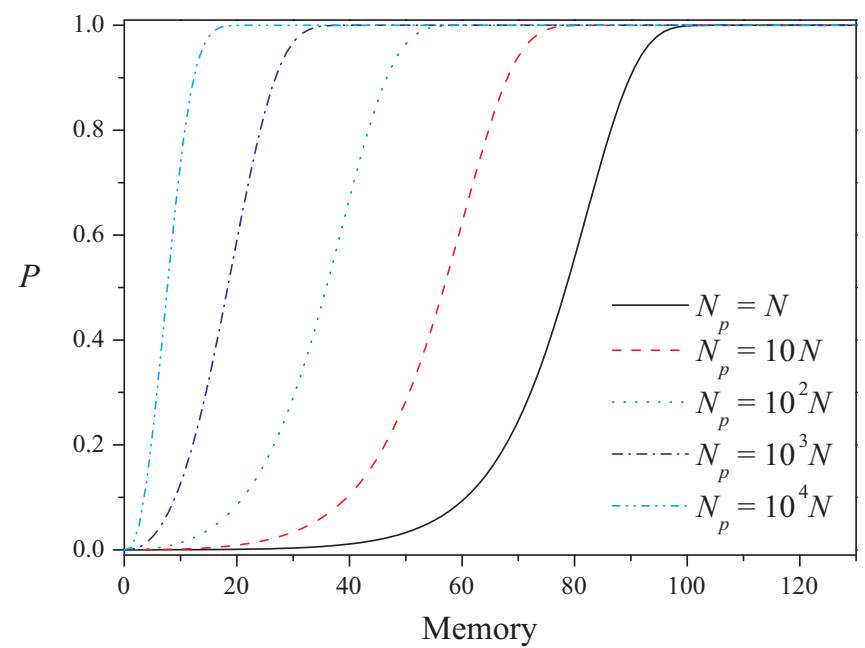

(a)

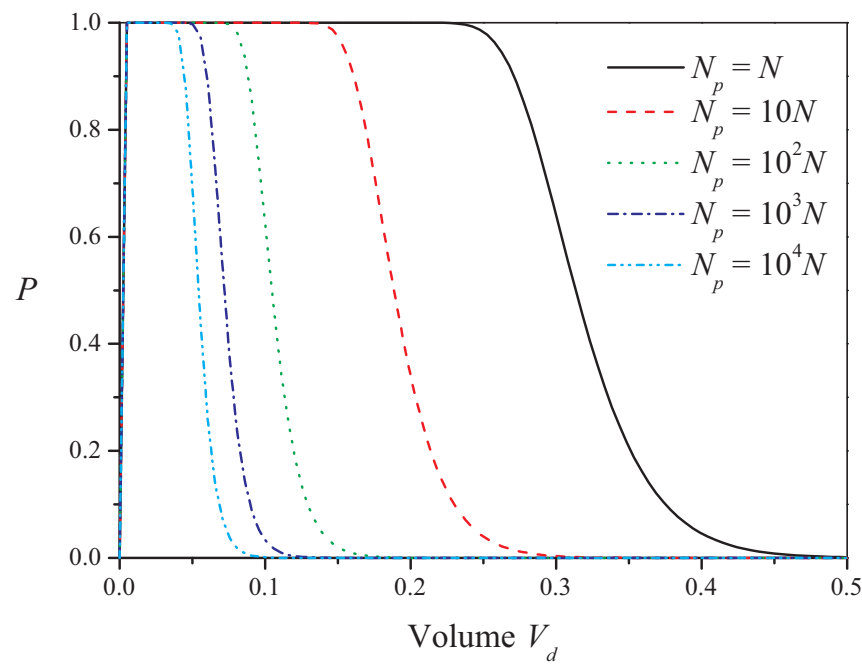

(b)

FIG. 5. (Color online) Death probability $P_{\Delta}$ (8) as function of (a) memory for volume $V_{d}=0.1$ and (b) volume $V_{d}$ for memory $\mu=75$, for $N=128$.

\section{B. Aging effect}

As a physical consequence due to the introduction of the variable $N_{p}$, the computational approach presents the aging effect. To analytically examine the aging effect in our model, we consider the walker survival probability after $N_{p}$ steps. Equation (7) gives the walker death probability at each step, so that the survival probability is $1-P_{\delta}$. After $N_{p}$ steps, the survival probability is $P_{s}=\left(1-P_{\delta}\right)^{N_{p}}$. Therefore, the probability of the walker death after $N_{p}$ steps is $P_{\Delta}=1-P_{s}$ :

$$
\begin{aligned}
& P_{\Delta}\left(N_{p}, V_{d}, \mu\right) \\
& \quad=1-\left\{1-\left(1-V_{d}\right)^{N}\left[-1+\left(1-V_{d}\right)^{-\mu}-\frac{\mu V_{d}}{1-V_{d}}\right]\right\}^{N_{p}} .
\end{aligned}
$$

The effect of increasing the exploration time $N_{p}$ is similar for any value of $N$. They present a sharp transition between the

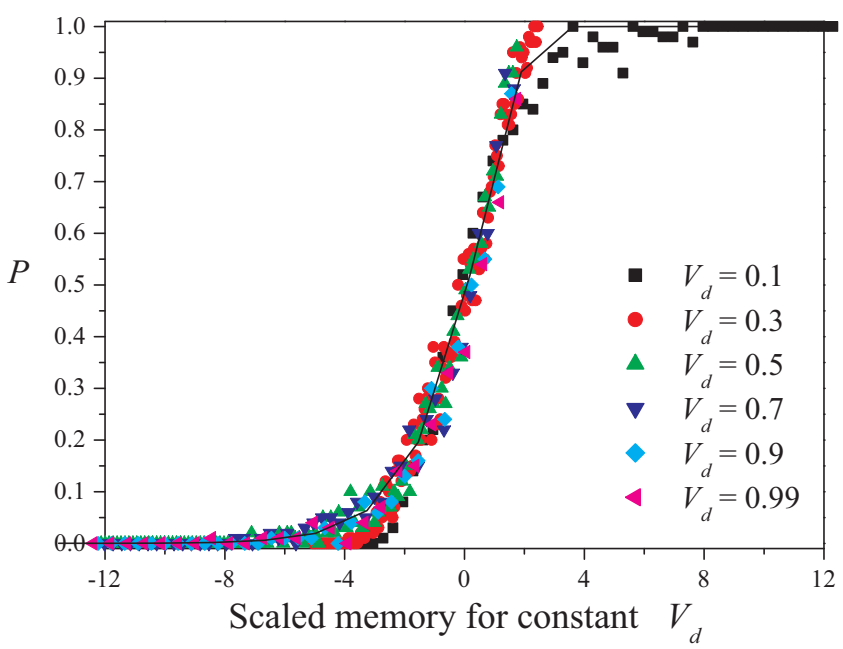

(a)

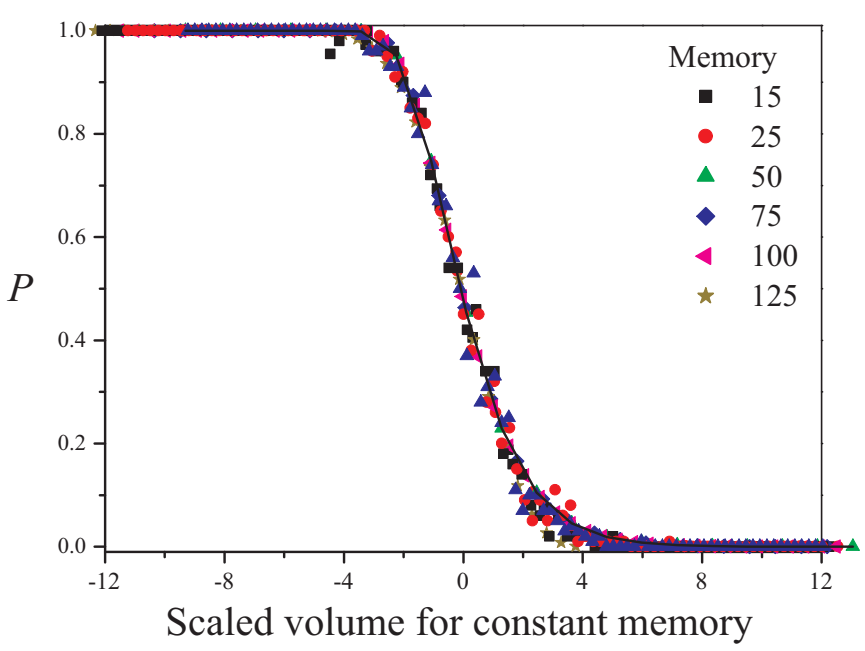

(b)

FIG. 6. (Color online) Collapse of death probability $P_{\Delta}$ as function of (a) scaled memory $\mu_{S}$ for constant volume $V_{d}$ and (b) scaled volume $V_{S}$ for constant memory $\mu$ for $N=128$ and $N_{p}=N$. Symbols stand for numerical data, and the continuous line for the analytical collapse. 
regions for the walker survival $\left(P_{\Delta} \approx 0\right)$ and death $\left(P_{\Delta} \approx 1\right)$. Further, $P_{\Delta}$ has a sigmoidal behavior as a function of both $\mu$ for a constant value of $V_{d}$ and $V_{d}$ for constant $\mu$.

The aging effect is shown in Figs. 5(a) and 5(b). Observe that the increasing of $N_{p}$ shifts the $P_{\Delta}$ curves. On one hand, for $V_{d}$ constant, the shift is towards small memory, and the survival is guaranteed only for small memory. On the other hand, for $\mu$ constant, the shift is towards larger volumes, since the survival occurs after a minimum value of $V_{d}$, for each $N_{p}$.

In addition, we have also analyzed $P_{\Delta}$ as a function of $\mu$ for different values of $V_{d}$, and as a function of $V_{d}$ for different values of $\mu$. Since we have similar curves, we rescale them to obtain a collapse. For the curves on a function of $\mu$ for constant $V_{d}$, from Eq. (8), we have calculated the inflection point, called a critical memory $\left(\mu_{c}\right)$, and the transition width $\left(w_{v, \mu}\right)$, a scale factor. Thus we obtained a scaled memory $\mu_{S}=\left(\mu-\mu_{c}\right) / w_{v, \mu}$ for constant volume $V_{d}$. We have done the same analysis for the curves for $V_{d}$ for constant $\mu$,

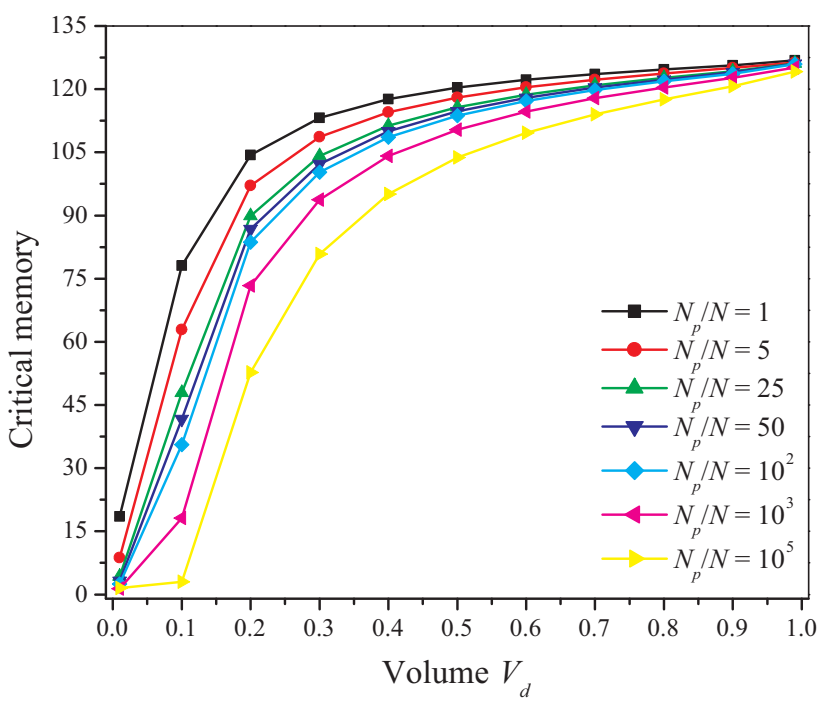

(a)

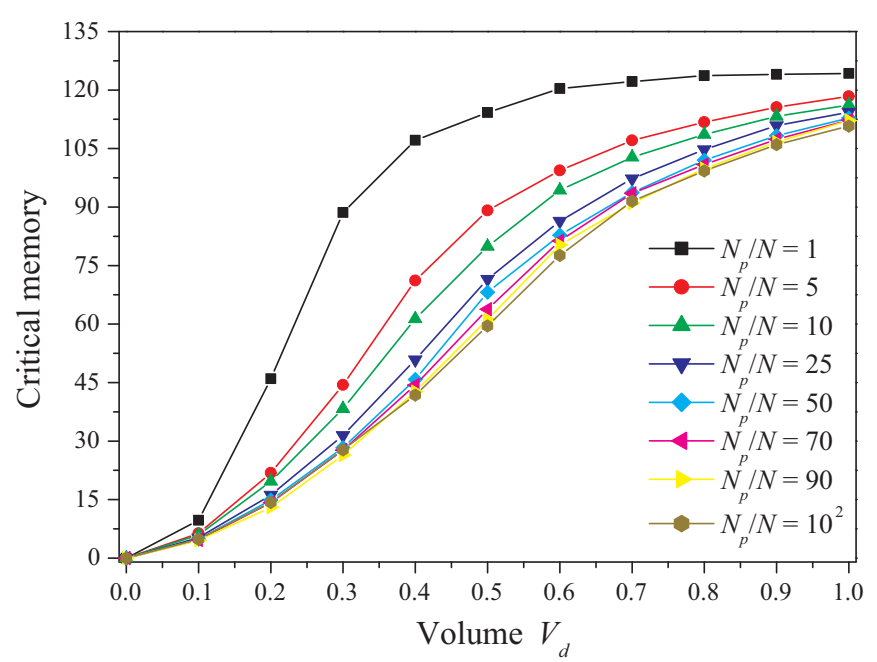

(b)

FIG. 7. (Color online) Critical memory $\mu_{c}$ as function of the volume $V_{d}$ for different values of $N_{p}$ for $N=128$ sites calculated (a) from the analytical curves and (b) from the numerical result. where the inflection point gives us the critical volume $V_{c}$, so the scaled volume is $V_{S}=\left(V_{d}-V_{c}\right) / w_{\mu, v}$. The collapses are shown in Fig. 6 with a comparison with computational results. The wide dispersion of the numerical data regarding the analytical prediction can be reduced by increasing the number of analyzed maps.

From our computational experiments, the parameter $N_{p}$, itself allows us to evaluate the aging effect of the system. Figure 6 shows that, as for the analytical case, there still is a way to collapse the data. This collapse coincides with the analytical case (continuous line). In addition, it shows that the transition curves behave quite similar with the analytical model. The values for the critical memory $\left(\mu_{c}\right)$ and the width of the transition region $\left(w_{v, \mu}\right)$ were obtained using the first and second numerical derivatives of each curve. In cases where the curve does not change the concavity, the concerning memory was obtained from the average height of $P_{\Delta}$ as $\mu_{c}$.

Plotting the values of $\mu_{c}$ as a function of $V_{d}$ for several values of $N_{p}$ it is possible to see the shift of the transition

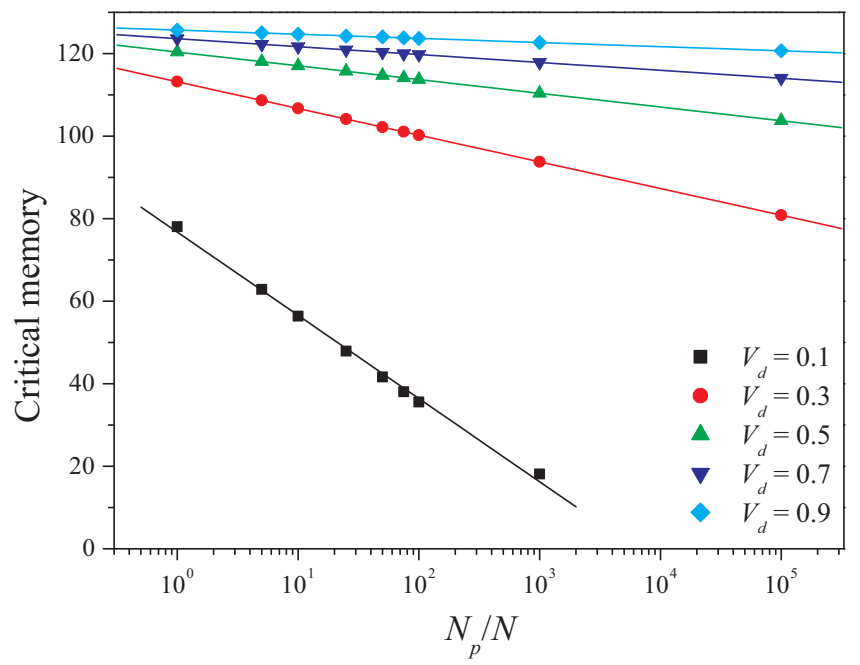

(a)

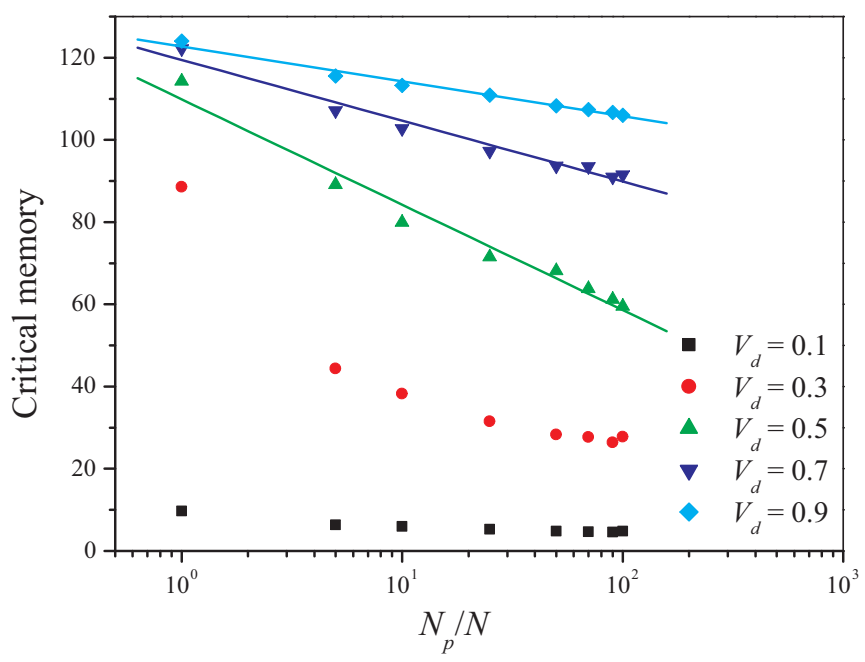

(b)

FIG. 8. (Color online) Critical memory $\mu_{c}$ for constant volume $V_{d}$ as function of $N_{p} / N$ for a system with $N=128$ sites obtained (a) from the analytical curves and (b) from the numerical results. 


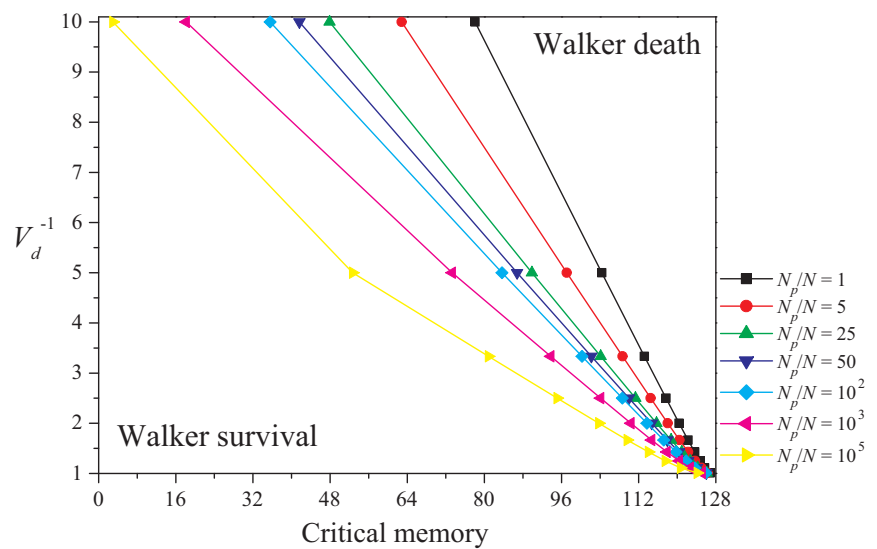

FIG. 9. (Color online) Crossover diagram for the parameters $V_{d}^{-1}$ and critical memory $\mu_{c}$. The curves discriminates the walker survival (below) and walker death (above) regions. The data points are given by analytical results.

curves, which is the main characteristic of the aging. Figure 7 shows the analytical and numerical shift on the transition region as a function of $N_{p}$ for $N=128$. Despite the difference between the predicted value, it is possible to see that in both cases, the behavior of the phenomenon of aging is the same: the reduction of the walker survival region as a function of $N_{p}$. In fact, this reduction is much faster in numerical experiments due to edge effects.

In our model, we understand the effect of the exploration time as a system aging effect. Since the value of $\mu_{c}$ decreases as exploration time increases, it could be possible to find a limit value for $N_{p}$, such that $\mu_{c}>0$. Thus, as in the onedimensional case of STW [21], we could obtain a value for $N_{p}$ after which the aging is slow and can be neglected. From Fig. 8, $\mu_{c}$ as function of $N_{p} / N$ for $N=128$ sites, one notes that $\mu_{c} \propto-\log \left(N_{p} / N\right)$ for any value of $V_{d}$, such that the the system suffers the aging effect until $N_{p} \rightarrow \infty$.

Although Fig. 8 shows that the numerical results are a bit different from the analytical results, it is possible to see that, for $V_{d} \geqslant 0,5$, the logarithmic behavior of the system aging effect remains the same. The slow aging for the case $V_{d}=0.1$ is due to the small generated volumes $V_{d}$, which have few sites in its interior, increasing the death probability. Thus, along with the edge effects, it causes death in a few steps, and therefore the transition memory is somewhat affected by the increasing on $N_{p}$.

Despite the fact numerical results quantitatively differ from the analytic results, they agree qualitatively with each other, showing similar behaviors for the analyzed quantities. The observed deviations are explained by edge effects that, for this particular case, are difficult to eliminate.

To summarize our results and to delineate the walker death and survival regions, we have plotted the crossover diagram, depicted in Fig. 9, for $V_{d}^{-1}$, which includes the dependence on the range $T$, and critical memory $\mu_{c}$. The region below the curves corresponds to the walker survival, and otherwise the walker death. As $N_{p} / N$ increases the walker death region increases. Namely, as the trajectory is longer, $n_{\mu}$ increases and $n_{v}$ decreases. Therefore, the probability of death in the next step increases. Note that an increase of $N_{p}$ can allow the crossover even in the thermodynamics limit. Further, for an environment with finite $N$, an increase of $N_{p}$ leads to an increase on the number of trajectories that end due to the walker death. Besides, in this case, death always occurs for $N_{p} \rightarrow \infty$.

\section{CONCLUSION}

We have considered a $d$-dimensional environment with $N$ randomly distributed sites. An agent explores this random medium moving stochastically within a range $T$ and a spatial memory $\mu$. The analytical results have been validated by numerical experiments and a crossover from the nonergodic to the ergodic regime has been found. With parameters fixed near this crossover, one can devise algorithms ensuring optimal search strategies. These algorithms may extend the agent deterministic moves used in pattern recognition, imaging texture analysis and classification, and complex network classification [26-30].

\section{ACKNOWLEDGMENTS}

J.M.B. acknowledges the financial support from the São Paulo Research Foundation FAPESP (Grant No. 2009/115676). R.S.G. (Grant No. 140420/2007-0) and ASM (Grant No. 305738/2010-0) acknowledge the Brazilian agency CNPq for support. The authors also acknowledge CAPES.
[1] D. Gale, J. Propp, S. Sutherland, and S. Troubetzkoy, Math. Intell. 17, 48 (1995).

[2] L. A. Bunimovich and S. E. Troubetzkoy, J. Stat. Phys. 67, 289 (1992).

[3] L. A. Bunimovich, Physica D 187, 20 (2004).

[4] H. K. Janssen and O. Stenull, Phys. Rev. E 85, 011123 (2012).

[5] G. F. Lima, A. S. Martinez, and O. Kinouchi, Phys. Rev. Lett. 87, 010603 (2001).

[6] H. E. Stanley and S. V. Buldyrev, Nature (London) 413, 373 (2001).

[7] H. Stanley, L. Amaral, X. Gabaix, P. Gopikrishnan, and V. Plerou, Physica A 299, 1 (2001).
[8] C. A. S. Terçariol and A. S. Martinez, Phys. Rev. E 72, 021103 (2005).

[9] J. M. Berbert and W. F. Fagan, Ecol. Complex. 12, 1 (2012).

[10] F. Bartumeus and J. Catalan, J. Phys. A 42, 434002 (2009).

[11] G. M. Viswanathan, V. Afanasyev, S. V. Buldyrev, E. J. Murphy, P. A. Prince, and H. E. Stanley, Nature (London) 381, 413 (1996).

[12] D. Boyer, O. Miramontes, and H. Larralde, J. Phys. A 43, 434015 (2009).

[13] D. Boyer, Eur. Phys. Lett. 83, 20001 (2008).

[14] D. Boyer, O. Miramontes, G. Ramos-Fernandez, J. L. Mateos, and G. Cocho, Physica A 342, 329 (2004). 
[15] D. Boyer, G. Ramos-Fernandez, O. Miramontes, J. L. Mateos, G. Cocho, H. Larralde, H. Ramos, and F. Rojas, Proc. R. Soc. Lond. B 273, 1743 (2006).

[16] M. C. Santos, D. Boyer, O. Miramontes, G. M. Viswanathan, E. P. Raposo, J. L. Mateos, and M. G. E. da Luz, Phys. Rev. E 75, 061114 (2007).

[17] C. A. S. Terçariol, R. S. González, and A. S. Martinez, Phys. Rev. E 75, 061117 (2007).

[18] C. A. S. Terçariol, R. S. González, W. T. R. Oliveira, and A. S. Martinez, Physica A 386, 678 (2007).

[19] S. Risau-Gusman, A. S. Martinez, and O. Kinouchi, Phys. Rev. E 68, 016104 (2003).

[20] A. S. Martinez, O. Kinouchi, and S. Risau-Gusman, Phys. Rev. E 69, 017101 (2004).

[21] J. M. Berbert and A. S. Martinez, Phys. Rev. E 81, 061127 (2010).

[22] S. Kirkpatrick, J. Stat. Phys. 34, 975 (1984).
[23] S. Kirkpatrick, C. D. Gelatt, and M. P. Vecchi, Science 220, 671 (1983).

[24] D. E. Goldberg, Genetic Algorithms in Search, Optimization and Machine Learning (Kluwer, Boston, 1989).

[25] W. Feller, An Introduction to Probability Theory and Its Applications (John Wiley \& Sons, New York, 1968), Vols. 1 and 2 .

[26] M. G. Campiteli, P. D. Batista, O. Kinouchi, and A. S. Martinez, Phys. Rev. E 74, 026703 (2006).

[27] A. R. Backes, W. N. Gonçalves, A. S. Martinez, and O. M. Bruno, Pattern Recogn. 43, 685 (2010).

[28] A. R. Backes, A. S. Martinez, and O. M. Bruno, Pattern Recogn. 44, 1684 (2011).

[29] W. N. Gonçalves, A. R. Backes, A. S. Martinez, and O. M. Bruno, Expert Sys. Appl. 19, 11818 (2012).

[30] W. N. Gonçalves, A. S. Martinez, and O. M. Bruno, Chaos 22, 033139 (2012) 